Annuaire suisse de politique de développement

26-2 | 2007

Financer le développement par la mobilisation des ressources locales

\title{
Gestion des finances publiques : une contribution à la bonne gouvernance financière
}

\section{Stefan Leiderer et Peter Wolff}

\section{CpenEdition}

\section{Journals}

Édition électronique

URL : https://journals.openedition.org/aspd/142

DOI : 10.4000/aspd. 142

ISSN : 1663-9669

Éditeur

Institut de hautes études internationales et du développement

Édition imprimée

Date de publication : 1 novembre 2007

Pagination : 175-195

ISBN : 978-2-88247-068-3

ISSN : 1660-5934

Référence électronique

Stefan Leiderer et Peter Wolff, « Gestion des finances publiques : une contribution à la bonne gouvernance financière », Annuaire suisse de politique de développement [En ligne], 26-2 | 2007, mis en ligne le 22 juin 2009, consulté le 21 septembre 2021. URL : http://journals.openedition.org/aspd/142 DOI : https://doi.org/10.4000/aspd.142 


\title{
Gestion des finances publiques: une contribution à la bonne gouvernance financière
}

\author{
Stefan Leiderer* et Peter Wolff**
}

\section{Introduction: la gestion des finances publiques comme élément de la bonne gouvernance financière}

L'expression gestion des finances publiques $(G F P)^{1}$ sert depuis quelques années de cadre à un débat animé sur les capacités et la réforme des finances publiques dans les pays en développement. L'importance de la GFP découle du rôle central que jouent les finances publiques dans un Etat démocratique désireux de se développer: une politique budgétaire axée sur des objectifs et une gestion budgétaire transparente sont à la base de prestations publiques visant à réduire la pauvreté et à atteindre les Objectifs du Millénaire pour le développement (OMD) dans les pays en développement. Pour ce qui est du contrôle des finances publiques, il compte parmi les tâches clés des parlements. La transparence des finances publiques de même que la participation de la population et de groupements de la société civile à l'établissement du budget témoignent du bon fonctionnement d'un système fondé sur l'obligation des autorités de rendre compte de leur action. A ce titre, des systèmes de GFP transparents et participatifs contribuent à faire respecter les droits humains, au sens d'autonomisation (empowerment). Ils permettent en effet aux citoyens d'influencer et d'adapter les milieux et les institutions politiques afin que ceux-ci servent leurs intérêts, de revendiquer leurs droits et de se faire entendre auprès des élites politiques. Puisque les divers éléments et soussystèmes abordés dans le débat sur la gestion des finances publiques ont une portée considérable, on tend désormais à parler plus largement de bonne gouvernance financière. On applique alors les principes de la bonne gouvernance au secteur des finances publiques (voir encadré 1).

* Stefan Leiderer est, depuis 2003, collaborateur scientifique au Deutsches Institut für Entwicklungspolitik (DIE) à Bonn (Allemagne). Il étudie en particulier les finances publiques dans les pays en développement et l'efficacité de nouveaux instruments de la coopération au développement.

** Peter Wolff ( $\mathrm{D}^{\mathrm{r}}$ ès sc. pol.) est collaborateur scientifique au Deutsches Institut für Entwicklungspolitik (DIE) à Bonn (Allemagne), où il dirige le département Economie mondiale et financement du développement. Il se consacre en priorité aux instruments et aux institutions de financement du développement aux niveaux national et international.

A l'exception de la conclusion, retravaillée par les auteurs pour le présent dossier, cet article reproduit un document de travail paru en allemand: Stefan Leiderer und Peter Wolff, Public Financial Management als Beitrag zu Good Financial Governance, Discussion Paper, n 10, Bonn, Deutsches Institut für Entwicklungspolitik, 2007 (N.D.E.).

1 En anglais public financial management (PFM). 


\section{Encadré 1: Principes de la bonne gouvernance appliqués aux finances publiques}

- Légitimité. Elle désigne la légitimation démocratique des décisions de politique financière. Elle englobe également une participation appropriée, équilibrée et non discriminatoire de la population (égalité entre femmes et hommes).

- Obligation de rendre compte. Ce principe comprend la responsabilité et l'obligation des instances étatiques de rendre compte aux citoyens de leurs actes et passe par la transparence sur les activités de l'Etat. L'efficacité et l'efficience de l'administration des finances publiques dépendent en effet de la possibilité dont bénéficient et usent la société et ses citoyens de demander des comptes à l'Etat.

- Légalité. Ce principe oblige la politique et l'administration financières publiques à respecter un cadre légal valable pour tous, équitable et impartial (obligation de l'administration de respecter le droit). La légalité des finances publiques garantit principalement leur prévisibilité et leur contrôle en toute fiabilité.

- Efficacité. Ce principe décrit la volonté et la capacité des organes et institutions publics d'accomplir leurs tâches. L'amélioration de l'efficacité dans le cadre de la bonne gouvernance financière passe avant tout par le renforcement des capacités des institutions publiques à gérer les ressources publiques.

- Action de l'Etat axée sur le développement. Ce principe se réfère aux valeurs fondamentales qui régissent l'activité étatique: justice sociale, durabilité écologique et économie de marché. Ces valeurs doivent transparaître non seulement dans les recettes, mais aussi dans les dépenses de l'Etat.

Source: Deutsche Gesellschaft für Technische Zusammenarbeit (GTZ), Good Financial Governance - Good Governance in Public Finance, Fiscal Studies, $n^{\circ}$ 3, Eschborn, 2006.

Depuis quelque temps, le débat international met avant tout l'accent sur l'importance d'une bonne gestion des finances publiques lorsqu'il est question des instruments du financement communautaire axé sur les programmes (FCAP). C'est compréhensible, car il faut, d'une part, un minimum de bonne gouvernance financière pour mettre efficacement ces instruments en œuvre; d'autre part, le recours au FCAP doit servir à stimuler la création ou l'amélioration des structures nécessaires à la gouvernance. Plus on confie l'exécution des FCAP aux systèmes de gestion des pays en développement - donc aussi à leurs propres systèmes budgétaires -, plus l'essentiel du travail de la politique de développement consistera à évaluer la qualité et l'efficacité de la GFP et à soutenir ces pays dans leurs efforts pour améliorer leur système budgétaire. Dans les pays en développement où la part du FCAP représente une grande partie de l'aide, les nouveaux instruments et structures de dialogue issus des principes de la Déclaration de Paris (appropriation, harmonisation et alignement) sont principalement axés sur les budgets des pays d'où provient une part croissante des contributions des donateurs.

Dans ce contexte, les réflexions présentées ici visent à fournir un aperçu du débat international en cours sur la GFP et contribuent à identifier les approches et les instruments qui permettraient à la coopération internationale de se positionner dans ce domaine, tant au niveau international que dans le cadre bilatéral.

\section{Qu'est-ce que la gestion des finances publiques?}

La GFP fait partie du système des finances publiques et, dans la pratique actuelle de la coopération internationale, elle se réfère principalement aux dépenses budgétaires (public expenditure management). Ce faisant, elle se concentre sur les 
instances et les procédures qui doivent garantir la transparence, l'efficacité et l'efficience des dépenses publiques, y compris dans l'utilisation de l'argent des donateurs. Cette concentration s'explique par l'existence des risques fiduciaires, qui découlent, aux yeux des donateurs, de l'implication des systèmes budgétaires des pays en développement dans la gestion de l'aide extérieure. Plus la gestion des finances publiques d'un pays est transparente et efficace, moins les risques fiduciaires paraissent élevés².

Parfaitement compréhensible du point de vue des donateurs, cet accent mis sur les dépenses et sur la gouvernance en matière de dépenses empêche toutefois de considérer toute la dimension de la gouvernance et des droits humains qu'englobe la bonne gouvernance financière. Voilà pourquoi nous commençons ici par replacer la gestion financière dans une perspective un peu plus large.

\section{La politique fiscale: un champ d'action transversal}

La notion de politique fiscale englobe les recettes et les dépenses publiques, ainsi que les stratégies et les instruments destinés à financer les déficits budgétaires. Traditionnellement, la coopération au développement s'intéressait avant tout aux effets macroéconomiques de la politique fiscale. Les programmes d'ajustement structurel du Fonds monétaire international (FMI) et de la Banque mondiale ont par exemple notamment imposé aux pays en développement des objectifs dont la réalisation devait leur conférer une grande stabilité macroéconomique. Si ces objectifs ont certes été atteints dans nombre de pays, c'est souvent au prix de profonds déficits structurels dans les budgets publics, de réductions des investissements publics et de coupes dans les dépenses importantes pour le développement. A long terme, ils ont profondément miné la croissance et la lutte contre la pauvreté.

Grâce à l'agenda des OMD, ainsi qu'à la volonté de mettre la croissance au service de lutte contre la pauvreté, la contribution que les finances publiques peuvent apporter au développement a regagné ses lettres de noblesse. En effet, le budget public n'est plus considéré en priorité comme un moyen de stabilisation macroéconomique, mais de plus en plus comme un instrument à même de favoriser la croissance et la réduction de la pauvreté ${ }^{3}$. L'élaboration de la politique fiscale consiste toujours davantage à analyser les diverses répercussions des recettes et des dépenses sur la croissance et sur la réduction de la pauvreté, et de les corriger si nécessaire. Considérés de ce point de vue, les déficits budgétaires et leur financement apparaissent sous un jour totalement différent, car il importe d'apprécier aussi s'ils soutiennent la croissance à plus long terme, par exemple en augmentant les investissements à même de stimuler le développement. Ce débat autour du rôle de l'espace fiscal en faveur de la croissance ou de la réduction de la pauvreté annonce inévitablement des corrections dans la politique restrictive menée par le FMI dans les pays en développement à faible revenu ${ }^{4}$.

Sur la définition des risques fiduciaires, voir plus bas l'encadré 3 (N.D.E.).

3 Poverty Reduction and Economic Management (PREM), Fiscal Policy for Growth and Development: An Interim Report, Washington, DC, The World Bank, 2006.

4 P. Heller, Understanding Fiscal Space, IMF Policy Discussion Paper, nº 05/4, Washington, DC, IMF, 2005. 
Les fondements de la politique fiscale d'un pays résident dans les options retenues en politique budgétaire: il s'agit de décider quelles tâches incombent avant tout à l'Etat et lesquelles seront mieux assumées par des acteurs non étatiques. De plus, il convient de choisir le mécanisme budgétaire, c'est-à-dire définir les modalités du financement des tâches étatiques, de même que leur répartition entre les instances centrales et les instances décentralisées. Ces dernières années, ces deux champs d'activités ont été marqués par de profonds changements dans nombre de pays en développement: d'une part, des activités traditionnellement assumées par l'Etat ont été privatisées; d'autre part, on observe une tendance à la décentralisation avec une redistribution des tâches entre acteurs publics. Vu l'extrême polarisation sociale, notamment dans certains pays latino-américains, il est d'autant plus crucial de miser sur la répartition territoriale des dépenses publiques et sur l'application d'instruments appropriés afin d'assurer l'équilibre financier, ainsi que sur l'accroissement des recettes et de la participation locale à l'élaboration des budgets.

Dans le même temps, l'agenda des OMD et l'amélioration des perspectives de croissance dans les pays pauvres ont conduit à une nouvelle perception stratégique de la politique budgétaire. La volonté d'assurer sa durabilité et sa prévisibilité pour ce qui est de la croissance et de la réduction de la pauvreté se reflète dans la planification financière à moyen terme, qui doit désormais aussi englober les contributions des donateurs, rarement prévisibles jusqu'ici. Les cadres de dépenses à moyen terme (CDMT) doivent définir l'allocation des ressources, aide extérieure comprise, de manière à garantir la mise en ouvre des stratégies de réduction de la pauvreté (SRP). Or, l'harmonisation des SRP et des CDMT compte parmi les défis que nul n'est encore parvenu à relever entièrement. La définition d'une politique budgétaire axée sur la pauvreté reste d'ailleurs théorique et son application demeure encore très vague. Pour remédier à ces lacunes, l'allocation des ressources pour lesquelles la budgétisation axée sur les résultats ne dispose pas d'instruments éprouvés (même dans la plupart des pays industrialisés) devrait s'orienter sur les objectifs et les programmes. On ignore en effet souvent les répercussions qu'une hausse ou une réallocation des ressources budgétaires dans certains domaines peut engendrer sur les résultats (de la réalisation des OMD, par exemple). Le respect des principes fondamentaux des droits humains - en particulier du droit à la participation, de l'égalité des chances et de la non-discrimination - permet pour le moins d'assurer que les préoccupations des pauvres soient prises en compte dans l'élaboration et l'administration du budget.

Outre les problèmes liés à la politique fiscale et budgétaire, l'administration des dépenses occupe aussi une place centrale dans l'application pratique. Du point de vue institutionnel et de la gouvernance, c'est le respect du droit qui revêt ici une grande importance. Dans quelle mesure le budget se fonde-t-il sur des bases légales et à quel point les diverses dépenses sont-elles conformes aux prescriptions légales? Les instruments permettant de garantir cette légalité comprennent un système budgétaire ordonné, une classification transparente des postes budgétaires de même qu'une administration budgétaire soigneusement organisée, en particulier au sein des différents ministères, auprès des autorités subordonnées et des bénéficiaires de contributions. Il importe par ailleurs de mettre en place un contrôle des dépenses publiques, qui permette de faire le lien entre la planification et l'application du budget. A cet effet, un nombre croissant de pays en 
développement se dotent de systèmes d'information spécifiques (systèmes d'information sur la gestion financière, FMIS).

Le contrôle des dépenses revêt une importance particulière dans la bonne gouvernance financière. Dans les pays industrialisés et la plupart des pays en développement, cette fonction relève des instances supérieures de contrôle (cour des comptes) et du parlement, les instances en question étant en principe responsables non pas devant le pouvoir exécutif, mais devant le législatif. Par ailleurs, dans les pays où ces instances n'assument pas leur tâche avec la fiabilité requise, ce sont les sociétés civiles qui prennent le relais, une presse libre constituant alors un appui décisif. Là où la reddition interne de comptes (responsabilité interne) manque de crédibilité aux yeux des donateurs, on renforce la reddition externe de comptes (responsabilité externe), notamment par le biais de systèmes de financement communautaire axé sur les programmes (FCAP). Ce renforcement est motivé d'une part par l'abaissement des risques fiduciaires et, d'autre part, par l'intérêt qu'il y a à promouvoir la bonne gouvernance financière. Les mécanismes de la responsabilité externe comprennent les revues régulières des dépenses publiques (PER, public expenditure reviews), que nombre de pays ont instituées pour vérifier les budgets annuels des ministères ainsi que le budget global de l'Etat. Les PER servent souvent de point de départ au dialogue sur le développement que le gouvernement entretient avec la communauté des donateurs au niveau des secteurs et des stratégies de réduction de la pauvreté (SRP), et auquel prennent part de plus en plus souvent les parlements et la société civile. Ces revues permettent d'harmoniser les objectifs budgétaires et la mise en œuvre de l'administration des dépenses. Viennent par ailleurs s'y ajouter les instruments diagnostiques présentés ci-après, qui servent à vérifier en détail la transparence et l'efficacité de l'administration des dépenses.

La politique et l'administration des recettes constituent également un domaine important de la politique fiscale. En matière de développement, l'encaissement de recettes pour financer les dépenses publiques ne revêt en effet pas moins d'importance que l'engagement de dépenses. Dès lors, la structure du système fiscal et le bon fonctionnement de l'administration fiscale exercent une influence déterminante sur la gouvernance et la réduction de la pauvreté. Seuls un système transparent et une bonne compréhension du principe de la légalité de l'administration - qui n'en est qu'à ses balbutiements dans maints pays - peuvent garantir le financement de tâches publiques par le prélèvement d'impôts. Dans le cadre du financement externe des budgets par le biais du FCAP, on peut enfin se demander s'il ne serait pas indiqué, par souci de durabilité, de faire dépendre les contributions externes de la mobilisation de ressources internes.

Pour conclure, à cause de l'accent mis sur les risques fiduciaires que court le donateur, la gestion des finances publiques se concentre essentiellement sur l'administration et le contrôle des dépenses. Une conception plus globale des finances publiques au service du développement ne devrait cependant jamais perdre de vue les interfaces avec d'autres secteurs de la politique fiscale. Pour respecter l'échéance des OMD, il est en effet crucial d'assurer le financement de tâches publiques (du côté des recettes) et de mettre en place une politique budgétaire à long terme axée sur la réduction de la pauvreté. 
Pour les diverses raisons présentées ci-dessus, l'efficacité de la gestion des finances publiques dans les pays en développement est au cœur du débat international sur l'augmentation des ressources destinées à la coopération au développement, sur l'efficacité et les risques de nouvelles formes de coopération (notamment les aides budgétaires), ainsi que sur le renforcement de la coordination et de l'harmonisation des activités des donateurs. Un vaste consensus s'est aujourd'hui établi autour de l'idée que pour assurer une mise en œuvre efficace des ressources de la coopération au développement, il importe de confier autant que possible leur gestion aux systèmes et aux structures des pays bénéficiaires. En adoptant la Déclaration de Paris sur l'efficacité de l'aide en mars 2005, les Etats donateurs et bénéficiaires se sont entre autres engagés à accroître le nombre des pays en développement dotés d'un véritable système de gestion des finances publiques et qui respectent, ou s'efforcent de respecter, les bonnes pratiques internationalement reconnues dans ce domaine ${ }^{5}$. Il n'en demeure pas moins que l'initiative et le pilotage des réformes nécessaires incombent aux pays bénéficiaires. De plus, les donateurs ont convenu de confier la mise en œuvre de leurs contributions aux systèmes nationaux de GFP dans les pays qui respectent déjà les bonnes pratiques en la matière ou ont lancé les programmes de réforme requis. A cet effet, les signataires de la Déclaration de Paris ont convenu de définir ensemble des échelles et des normes, afin de mesurer l'efficacité et le niveau de responsabilité des systèmes de GFP.

Au-delà de ces exigences politiques reposant sur une vaste assise internationale, le débat autour des réformes de la gestion des finances publiques (GFP) dans les pays en développement est dominé par quelques rares acteurs, dont notamment la Banque mondiale, qui a multiplié ses capacités dans ce domaine au cours de ces dernières années. Outre les départements et groupes de travail spécifiques de la Banque mondiale, c'est surtout le secrétariat du PEFA (Public Expenditure and Financial Accountability/Dépenses publiques et responsabilité financière), financé par plusieurs pays donateurs et également sis auprès de la Banque mondiale, qui dirige le débat sur l'évaluation et la réforme des systèmes de GFP dans les pays en développement. Une Activité conjointe sur la gestion des finances publiques a de plus été créée dans le cadre du Comité d'aide au développement (CAD) de l'Organisation de coopération et de développement économiques (OCDE), afin de renforcer l'application de la Déclaration de Paris et de promouvoir les échanges de savoir-faire et d'expériences en matière de GFP entre donateurs et pays partenaires. Une table ronde du CAD traitant de la passation des marchés se penche en outre sur les questions de transparence et de bonnes pratiques en matière d'attribution de mandats publics. Enfin, le Réseau du CAD sur la gouvernance (GOVNET) a fait du thème taxation et responsabilité la priorité de son programme 2007-2008 et mis sur pied un groupe de travail qui explorera ce domaine particulier.

5 Outre l'expression d'une volonté politique, la Déclaration de Paris comprend des indicateurs chiffrés pour mesurer les progrès de la gestion financière. D'ici 2010, la moitié des pays partenaires doivent progresser d'une unité (soit de 0,5 point) sur l'échelle GFP/EPIN (évaluation de la politique et des institutions nationales) de la Banque mondiale. 
Parmi les donateurs bilatéraux, le Royaume-Uni exerce, en particulier par l'octroi ciblé de moyens destinés à la recherche, une grande influence sur le débat autour de la gestion des finances publiques.

\section{Evaluation de l'efficacité de systèmes de gestion des finances publiques}

Après l'adoption de la Déclaration de Paris, le PEFA a élaboré l'approche renforcée à l'appui de la réforme des systèmes de gestion des finances publiques, afin de délimiter un cadre de référence internationalement reconnu servant à simplifier l'évaluation et la réforme des finances publiques dans les pays en développement. Cette approche se fonde sur un cadre de mesure de la performance de la gestion des finances publiques, tracé par un groupe de travail sur la GFP réunissant des représentants du PEFA, de la Banque mondiale et du FMI. Ce cadre évalue l'efficacité de la gestion des finances publiques d'un pays en se fondant sur une liste de 31 indicateurs $^{6}$ définis pour les six grandes dimensions de la GFP (voir encadré 2).

\section{Encadré 2: Dimensions de l'efficacité de la gestion des finances publiques selon le PEFA}

ـ Crédibilité du budget. Le budget est réaliste et il est exécuté comme prévu.

- Exhaustivité et transparence. Le budget et le suivi des risques budgétaires sont exhaustifs et les informations financières et budgétaires sont accessibles au public.

- Budgétisation fondée sur les politiques nationales. Le budget est établi en tenant dûment compte des politiques publiques.

- Prévisibilité et contrôle de l'exécution du budget. Le budget est exécuté d'une manière ordonnée et prévisible, et des mécanismes existent pour assurer le contrôle et le suivi de l'utilisation des fonds publics.

- Comptabilité, enregistrement de l'information et rapports financiers. Des données et des informations appropriées sont produites, conservées et diffusées pour les besoins de prises de décision, de gestion et de préparation des rapports.

- Surveillance et vérification externes. Les dispositions relatives à l'examen des finances publiques et aux mesures de suivi par les responsables concernés sont opérationnelles.

Source: Dépenses publiques et responsabilité financière (PEFA), Cadre de mesure de la performance de la gestion des finances publiques, Washington, DC, Banque mondiale, 2005.

Le cadre de mesure de la performance ne doit pas servir en premier lieu à évaluer les risques, mais à créer des bases transparentes et aussi objectives que possible, afin d'instaurer un vaste dialogue entre donateurs et gouvernements partenaires sur l'élaboration de programmes stratégiques pour réformer la GFP.

L'objectif initial, visant à utiliser le cadre de mesure de la performance pour remplacer d'autres instruments diagnostiques, tels que l'évaluation de la responsabilité financière (CFAA), les rapports analytiques sur la passation des marchés (CPAR) et les revues régulières des dépenses publiques (PER), a été

6 Trois de ces indicateurs servent à évaluer les activités des donateurs qui exercent une influence sur l'efficacité de la gestion des finances publiques. 
abandonné ${ }^{7}$. Les évaluations du PEFA doivent bien plus servir à identifier les domaines dans lesquels s'imposent des analyses fouillées et un dialogue approfondi en vue de formuler des stratégies de réforme appropriées ${ }^{8}$.

\section{Encadré 3: Risques fiduciaires et risques relevant de la politique de développement}

En général, on considère la gestion des finances publiques (GFP) sous deux angles: celui du développement et celui des risques. Pour ce qui est du développement, la GFP est perçue comme une dimension essentielle de la gouvernance et un instrument qui détermine l'efficacité de la coopération au développement et des efforts des pays en développement eux-mêmes. Parmi les risques, on considère surtout les risques fiduciaires de certaines formes d'aide au développement (les aides budgétaires en particulier), ces risques découlant des faiblesses de la GFP dans le pays bénéficiaire. Pour ce qui est de savoir s'il est possible de distinguer vraiment les risques fiduciaires des risques liés à la politique de développement, les avis divergent. Cette distinction s'avère en réalité moins cruciale pour l'évaluation des risques que pour l'élaboration de stratégies visant à les contrer efficacement. Le principal défi auquel la coopération est confrontée en matière de GFP est d'assurer un traitement approprié de ces deux types de risque dans un processus lancé par les partenaires.

Selon le Département britannique pour le développement international (DFID), le risque fiduciaire dans la mise en œuvre de ressources destinées à la coopération au développement revêt trois aspects:

- les ressources ne sont pas utilisées pour réaliser l'objectif prévu;

口 elles ne sont pas mises en œuvre de manière économique, efficace et efficiente loptimisation des ressources):

口 les informations sur leur utilisation ne sont pas suffisantes.

Pour évaluer les risques, le DFID se fonde sur 12 règles de bonne pratique et sur 15 repères en matière de GFP qui en découlent. Ceux-ci servent en particulier aussi à apprécier si le système de GFP est suffisamment axé sur la réduction de la pauvreté. Le DFID évite donc d'établir une distinction nette entre risques fiduciaires et risques liés à la politique de développement. A l'inverse, la Banque mondiale préconise une notion plus stricte, selon laquelle les risques fiduciaires ne relèvent que de la gestion des finances. Dans ce cas, il s'agit donc uniquement d'apprécier si les ressources mises à disposition alimentent effectivement le budget et si elles sont, à ce titre, administrées de manière appropriée.

L'appréciation des risques fiduciaires par les donateurs varie parfois grandement selon que ceux-ci mettent l'accent par exemple sur le respect de normes ou plutôt sur l'existence d'efforts crédibles destinés à améliorer la GFP. Nul ne conteste cependant que, dans l'idéal, tous les donateurs devraient se fonder sur une seule et même base d'informations pour procéder à leur évaluation (ce qui ne les empêchera pas de parvenir à des résultats différents).

Sources: Department for International Development (DFID), Managing Fiduciary Risks When Providing Poverty Reduction Budget Support, DFID Briefing, London, DFID, 2004. D. Shand, "Managing Fiduciary Issues in Budget Support Operations", in S. Koeberle, Z. Stavreski, and J. Walliser, Budget Support as More Effective Aid? Recent Experiences and Emerging Lessons, Washington, DC, The World Bank, 2006, pp. 27-44.

7 Pour un aperçu des instruments diagnostiques de la GFP, voir: S. Leiderer, Analyse und Reform des öffentlichen Budgetmanagements: Ein zentrales Querschnittsthema für die programmorientierte Entwicklungspolitik, Analysen und Stellungnahmen, $\mathrm{n}^{\circ} 3 / 2005$, Bonn, Deutsches Institut für Entwicklungspolitik (DIE), 2005.

8 Dans nombre de pays, des PER régulières, pilotées par le gouvernement lui-même, font désormais partie intégrante du processus ordinaire de la planification et de la gestion financières. 


\section{Evaluation de la transparence et de la participation}

La transparence de toutes les étapes du cycle budgétaire et la possibilité de prendre part au processus budgétaire sont les composantes fondamentales de la bonne gouvernance financière. L'encadré 4 présente le code de bonnes pratiques formulé par le FMI dans le but de promouvoir la transparence des finances publiques.

\section{Encadré 4:}

\section{Code de bonnes pratiques du FMI en matière de transparence des finances publiques}

ـ Le secteur de l'administration publique doit être clairement séparé du reste de l'économie, et la répartition des attributions entre politique et administration (ou décision et gestion) doit être clairement définie.

- La gestion des finances publiques doit s'inscrire dans un cadre juridique et administratif clair.

ـ Le public doit être pleinement informé de l'activité financière passée, présente et prévue de l'administration publique.

ـ Le service public doit s'engager à diffuser en temps voulu des informations sur les finances publiques.

- La documentation budgétaire doit préciser les objectifs en matière de finances publiques, le cadre macroéconomique, les politiques sur lesquelles repose le budget et les principaux aléas budgétaires identifiables.

ـ Les informations budgétaires doivent être présentées de manière à satisfaire aux besoins de l'analyse des politiques et de la clarté des comptes.

ـ Les modalités d'exécution et de suivi des dépenses approuvées doivent être clairement définies.

$\checkmark$ Des rapports complets et fiables doivent être publiés dans les délais prévus et mettre en évidence les tendances dans les finances publiques.

ـ Les informations relatives aux finances publiques doivent faire l'objet d'un examen public et indépendant.

Source: Deutsche Gesellschaft für Technische Zusammenarbeit (GTZ), Good Financial Governance - Good Governance in Public Finance, Fiscal Studies, nº 3, Eschborn, GTZ, 2006.

Soumettre véritablement les processus budgétaires à la surveillance et au contrôle des législatifs et du public n'est toutefois pas seulement indispensable pour accroître l'efficacité et l'efficience de la planification budgétaire et de l'allocation des ressources par l'exécutif (dans la mesure où ces contrôles renforcent l'obligation des gouvernements de rendre compte), mais contribue aussi directement au respect des droits civiques et humains (notamment du droit à la participation aux processus politiques) au sens d'une autonomisation ${ }^{9}$. Voilà pourquoi on tend à percevoir de plus en plus l'analyse budgétaire et la promotion des finances publiques comme des éléments importants des efforts menés par la société civile en vue de renforcer l'obligation des gouvernements de rendre compte de leur action ${ }^{10}$ et de promouvoir les droits humains ${ }^{11}$. Les groupements

9 A. Norton and D. Elson, What's behind the Budget? Politics, Rights and Accountability in the Budget Process, London, Overseas Development Institute (ODI), 2002.

10 M. Robinson, Budget Analysis and Policy Advocacy: The Role of Non-governmental Public Action, IDS Working Paper, $n^{\circ}$ 279, Brighton, Institute of Development Studies (IDS) at the University of Sussex, 2006, p. 8.

11 FUNDAR - Centro de Análisis e Investigación, International Budget Project (IBP), and International Human Rights Internship Program (IHRIP), Dignity Counts: A Guide to Using Budget Analysis to Advance Human Rights, México, FUNDAR; Washington, DC, IBP; IHRIP, 2004, p. 2. 
de la société civile ne pourront toutefois prendre pleinement part aux processus budgétaires que s'ils disposent d'informations complètes, compréhensibles et d'actualité sur les finances publiques ${ }^{12}$.

Dans ce contexte, le Projet budgétaire international (International Budget Project, IBP $)^{13}$ du Center on Budget and Policy Priorities, à Washington, a élaboré, en marge de l'Initiative sur le budget ouvert 2006, une procédure basée sur un questionnaire, qui permet d'évaluer la transparence et la participation dans les systèmes de GFP, et de les classer selon un index sur le budget ouvert $^{14}$. Cet instrument est utile à double titre. Premièrement, l'identification des informations budgétaires rendues publiques dans les pays en développement devrait renforcer les capacités des groupements de la société civile de prendre une part active à l'analyse des finances publiques. Deuxièmement, la publication de tous les systèmes de GFP classés selon leur transparence devrait pousser les gouvernements à soutenir les groupements de la société civile qui s'activent dans le domaine de la gestion des finances publiques ${ }^{15}$.

\section{Réformes de la gestion des finances publiques}

Le principal défi que la politique de développement se doit de relever pour renforcer durablement les systèmes nationaux de GFP consiste moins à identifier simplement les grandes lacunes de ces systèmes qu'à formuler des réformes réalistes et efficaces, puis à les appliquer avec le soutien approprié des donateurs. Or, tous les acteurs ont encore beaucoup à apprendre dans ce domaine. Même l'approche renforcée à l'appui de la réforme des systèmes de gestion des finances publiques du PEFA ne fournit pas encore de solutions pratiques, qui sortent complètement du cadre théorique, afin de formuler des stratégies et des programmes de réforme réalistes et adaptés aux évaluations des systèmes de GFP. Des stratégies de réforme menées avec succès permettent néanmoins aux milieux internationaux de recenser quelques bonnes pratiques ou leçons tirées. Un large consensus s'est ainsi établi autour du fait que la réforme du système de gestion des finances publiques est un processus à long terme (certains donateurs jugent réaliste de prévoir quinze à vingt-cinq ans pour la mener à bien) et extrêmement politisé, qui bouleverse les structures du pouvoir et la répartition des ressources dans un pays. Au sein de la communauté des donateurs, on admet par ailleurs de plus en plus que le succès des stratégies de réforme de la GFP ne dépend pas uniquement du contenu des réformes, mais avant tout de leurs modalités ${ }^{16}$.

12 International Budget Project (IBP), Guide pour répondre au questionnaire sur le budget ouvert. Explicitation des questions et des modalités de réponse, Washington, DC, IBP, 2005, p. 3.

13 <http://www.internationalbudget.org >.

$14<$ http://www.openbudgetindex.org $>$.

15 Cet objectif distingue l'Initiative sur le budget ouvert des premiers instruments destinés à évaluer la transparence des finances publiques, tel le rapport du FMI sur l'observation des normes et des codes (Report on the Observance of Standards and Codes), qui sont avant tout axés sur les besoins d'information des donateurs (voir: S. Leiderer, Öffentliches Budgetmanagement in Entwicklungsländern: Analyseinstrumente und Ansatzpunkte der programmorientierten Entwicklungszusammenarbeit, Berichte und Gutachten, no 7/2004, Bonn, Deutsches Institut für Entwicklungspolitik [DIE], 2004).

16 Voir: Deutsche Gesellschaft für Technische Zusammenarbeit (GTZ), Good Financial Governance Good Governance in Public Finance, Fiscal Studies, n 3, Eschborn, GTZ, 2006, p. 13. Dépenses publiques et responsabilité financière (PEFA), Etude des mesures utilisées pour remédier aux lacunes des systèmes de gestion des finances publiques dans le contexte d'un programme d'appui de réformes, Washington, DC, Banque mondiale, 2003. 
Voilà pourquoi il s'avère impossible de piloter efficacement les réformes de la GFP de l'extérieur. En effet, ces réformes doivent résulter d'un processus endogène, défini par les instances politiques et institutionnelles, dont l'initiative, l'application et le suivi incombent en premier lieu aux pays partenaires. L'appropriation et le leadership des partenaires revêtent dès lors une importance décisive pour le succès de ces réformes. Quant à la coopération au développement, son rôle consiste avant tout à susciter la réflexion, à appuyer la formulation de stratégies de réforme dans le cadre du dialogue avec les partenaires et à fournir un soutien financier et technique à leur application. Les efforts de réforme doivent avant tout viser à développer les capacités des pays partenaires en matière de gestion des finances publiques. A cet effet, le CAD (OCDE) a formulé des principes directeurs pour renforcer les capacités en matière de gestion des finances publiques (voir encadré 5).

\section{Encadré 5: Principes directeurs du CAD pour renforcer les capacités en matière de gestion des finances publiques}

ـ Favoriser la conduite des opérations et l'appropriation par le pays partenaire doit être une préoccupation première des donateurs.

- La conception et l'organisation dans le temps du développement des capacités ne doivent pas refléter des solutions standard ou importées, mais s'adapter aux spécificités du pays.

- Les niveaux (institutions, organisations et individus) auxquels un renforcement des capacités s'impose, avec leurs aspects techniques et managériaux, doivent tous être pris en compte dans la conception et la mise en œuvre des programmes.

- Les donateurs doivent veiller à la cohérence et à la coordination de leur soutien et adopter une approche fondée sur les programmes.

Source: CAD, Harmoniser l'aide pour renforcer son efficacité, vol. 2: Le soutien budgétaire, les approches sectorielles et le développement des capacités en matière de gestion des finances publiques, Lignes directrices et ouvrages de référence du CAD, Paris, OCDE, 2003

En général, quelle que soit l'approche choisie pour réformer la GFP, il faut se rappeler que la structure et le fonctionnement des systèmes des finances publiques sont liés au contexte et peuvent donc varier grandement d'un pays à l'autre. Il serait dès lors vain de vouloir définir un modèle standard de réforme de la GFP, et il serait parfaitement impossible de transposer sans autre les composants éprouvés d'un système budgétaire d'un pays dans un autre, car le fonctionnement de certains secteurs de la GFP dépend des caractéristiques du système dans son ensemble. Des mesures isolées visant à améliorer certains éléments de la GFP n'ont dès lors guère de chances de succès et peuvent même s'avérer contreproductives. Pour être efficaces, les réformes doivent donc toujours tenir compte des spécificités du pays concerné et viser l'ensemble de sa gestion des finances publiques ${ }^{17}$. Pour cette même raison, la conception stratégique des processus de réforme, notamment la planification des diverses étapes de la réforme dans le temps, revêt une importance particulière ${ }^{18}$. Il faut par exemple compter des années

17 S. Leiderer, Analyse und Reform des öffentlichen Budgetmanagements: Ein zentrales Querschnittsthema für die programmorientierte Entwicklungspolitik, op. cit., p. 4.

18 Voir: Dépenses publiques et responsabilité financière (PEFA), Etude des mesures utilisées pour remédier aux lacunes des systèmes de gestion des finances publiques dans le contexte d'un programme d'appui de réformes, op. cit. 
pour achever des réformes institutionnelles et développer durablement les capacités alors qu'il peut s'avérer indispensable d'obtenir des succès visibles à court terme afin de préserver l'élan et l'appui politique du processus de réforme.

\section{Le besoin de connaissances en matière de gestion des finances publiques}

Comme les réformes de la GFP impliquent des processus aussi techniquement complexes que politiquement sensibles, les donateurs doivent s'attacher à coordonner et à harmoniser leurs activités pour soutenir de telles réformes dans les pays partenaires. La réforme de la GFP constitue dès lors une tâche commune à laquelle doivent s'atteler tous les acteurs du développement. Compte tenu des objectifs adoptés dans la Déclaration de Paris, la plupart des pays en développement ont entrepris de mieux intégrer les activités des partenaires et des donateurs dans le domaine de la GFP et de les mettre en œuvre en recourant à des mécanismes de financement communs (en particulier les aides budgétaires sans affectation précise et les fonds communs). Les aides isolées visant à optimiser la GFP afin d'améliorer la coordination et l'alignement passent de plus en plus au second plan, au profit de programmes conjoints, définis d'entente avec le gouvernement partenaire et destinés à soutenir les réformes de la GFP ${ }^{19}$.

\section{Encadré 6 : Aides budgétaires et réforme de la gestion des finances publiques}

Décharger les capacités administratives et managériales des pays partenaires par des aides financières communes des donateurs augmente sensiblement l'efficacité de la coopération au développement. Les aides budgétaires ne constituent cependant pas une contribution directe à l'amélioration des systèmes de GFP dans les pays bénéficiaires. L'effet d'allègement ne sera en effet perceptible que si deux conditions sont remplies: premièrement, les ressources de l'aide publique au développement doivent représenter une part importante des dépenses publiques nationales; deuxièmement, une proportion substantielle de ces ressources doit être fournie sous forme d'aide budgétaire. Lorsque ces deux conditions sont déjà remplies, un transfert au profit des aides budgétaires peut notamment contribuer à accroître la portée des effets positifs de l'optimisation de la GFP, et augmenter d'autant l'efficacité et l'efficience d'une plus grande partie des dépenses publiques. Au-delà de leur effet d'allègement, les aides budgétaires et d'autres formes de financement communautaire axé sur les programmes (FCAP) peuvent concourir par d'autres biais à optimiser la GFP. D'un côté, il est possible d'utiliser directement des ressources du FCAP pour financer des mesures destinées à améliorer la GFP. De l'autre, ces mesures renforcent le rôle des finances publiques en tant qu'instrument clé de l'élaboration des politiques et peuvent, en modifiant les rapports de pouvoir, produire un impact positif sur l'efficacité de l'action étatique, en renforçant par exemple la position du Ministère des finances face aux autres ministères. Les contributions non financières dans le cadre du FCAP jouent cependant un rôle crucial dans le renforcement de la GFP. Elles comprennent en particulier le débat sur la GFP ainsi que le dialogue entre donateurs et gouvernement partenaire, de même qu'entre d'importants acteurs nationaux, ce qui met l'efficacité du système de gestion des finances publiques au centre du débat et incite le gouvernement à rendre publiques les informations sur sa gestion budgétaire. Ce progrès peut à son tour ménager d'appréciables marges de manœuvre aux partisans de la réforme.

Sources: T. Williamson, «General Budget Support and Public Financial Management Reform: Emerging Lessons from Tanzania and Uganda ", in S. Koeberle, Z. Stavreski, and J. Walliser, Budget Support as More Effective Aid? Recent Experiences and Emerging Lessons, Washington, DC, The World Bank, 2006, pp. 139-152. International Development Department (IDD) and Associates, Evaluation of General Budget Support: Synthesis Report, Birmingham, University of Birmingham, 2006.

19 Dans la plupart des pays en développement, l'efficacité de la coopération technique et du renforcement des capacités en matière de GFP demeure cependant compromise par l'éparpillement des activités et le manque de coordination. 
Telle qu'elle est décrite, l'approche renforcée du PEFA n'offre pas la même adéquation pour tous les pays. Elle convient en particulier aux pays dépendant beaucoup de l'aide internationale, disposant de capacités administratives relativement réduites, mais dotés d'institutions démocratiques appropriées et d'un minimum de bonne gouvernance ${ }^{20}$. D'autres approches sont en principe nécessaires tant pour les pays extrêmement faibles et fragiles que pour ceux dotés d'institutions et de capacités bien développées. Cette remarque s'applique toutefois moins à l'évaluation ${ }^{21}$ qu'au choix de l'approche appropriée de la réforme. Dans les pays fragiles, les donateurs doivent en général assumer plus de responsabilités et piloter davantage le processus de réforme de la GFP, afin de ne pas surcharger les maigres capacités des partenaires. A l'inverse, les pays dotés d'institutions efficaces et de capacités administratives bien développées seront mieux à même d'identifier et de coordonner les aides adéquates provenant des donateurs. De tels pays ont en général moins besoin d'un large appui financier pour soutenir les réformes de la GFP que d'un transfert de connaissances techniques dans les divers secteurs de la GFP.

\section{Encadré 7: Renforcement des capacités dans les Etats fragiles}

La notion d' «Etats fragiles» désigne des types de pays fort différents, allant des nations en situation de postconflit aux régimes chroniquement faibles, même en l'absence de conflit, ou menacés d'effondrement. La variété des conditions socioéconomiques et politiques qui y règnent s'avère donc tout aussi riche. Aux yeux du Réseau du CAD sur la gouvernance (GOVNET), c'est justement dans ces pays que le renforcement des capacités revêt une importance capitale. Pour renforcer les Etats fragiles, on ne peut toutefois pas s'appuyer sur un modèle standard, car il faut prendre en considération les particularités de chacun d'entre eux. On peut néanmoins dégager des principes généraux et des expériences en matière de développement des capacités. Mentionnons en particulier le besoin d'opérer un choix très sélectif parmi les domaines d'intervention et les instruments à mettre en œuvre. En tête des priorités, il faut placer les fonctions clés de l'administration publique, dont le renforcement peut réduire la fragilité de l'Etat, puisqu'il lui confère les capacités d'offrir un minimum de prestations à ses citoyens. Or, la gestion des finances publiques compte parmi ces fonctions clés car elle permet de garantir à court terme la sécurité et la fourniture de prestations de base, et elle crée à moyen terme les conditions requises pour assurer toute la gamme des prestations publiques. Parmi les autres leçons tirées, le CAD mentionne celles-ci:

ـ lorsque la capacité étatique est faible mais la volonté politique bien présente, les efforts de renforcement des capacités devront se concentrer sélectivement sur les fonctions essentielles de l'Etat, afin de lui assurer au moins un minimum d'efficacité dans son rôle à l'égard des citoyens;

- les outils de planification mis au point pour être utilisés après des conflits, comme la «matrice de résultats pour la transition ", peuvent être utiles en vue d'intégrer le soutien en faveur du renforcement des capacités;

20 Les avis des donateurs sur les instruments à mettre en œuvre dans les divers types de pays varient sensiblement. Tandis que certains partent de l'idée que les aides budgétaires ne doivent être accordées qu'à des pays possédant une GFP relativement efficace, d'autres pensent qu'il faut considérer les aides budgétaires comme des instruments propres à apporter un appui solide justement aux pays fragiles et ne possédant que de faibles capacités en matière de GFP, afin de les aider à réformer durablement leur systèmes de gestion et de renforcer les capacités qui en ont besoin.

21 En principe, le cadre de mesure de la performance est à même de s'adapter si bien aux différents types de pays qu'il suffit de retenir, parmi la série d'indicateurs qu'il fournit, ceux qui sont les mieux adaptés au contexte. Si l'on veut que les évaluations restent comparables, il serait cependant peu judicieux d'adapter les critères d'évaluation. 
- le soutien extérieur du renforcement des capacités devrait respecter le principe du changement endogène et de l'encouragement du pays à prendre la direction des opérations, même lorsque les conditions matérielles semblent s'y opposer;

- il importe que les nouvelles initiatives en matière de renforcement des capacités n'érodent pas ou ne fassent pas double emploi avec les capacités existantes, en termes individuels, organisationnels ou d'environnement général;

口 dans d'autres cas, la sélectivité sectorielle, ou «alignement partiel», peut produire des retombées stratégiques;

- même dans les Etats qui ont de sérieux problèmes de gouvernance, un renforcement modeste des capacités demeure possible.

Source: Réseau du CAD sur la gouvernance, Relever le défi posé par le renforcement des capacités. Evoluer vers de bonnes pratiques, Paris, OCDE, 2006.

Les contributions non financières s'avèrent cependant déterminantes pour aider les partenaires à développer leurs capacités en matière de GFP et à mener les réformes à bien, même dans les pays où l'approche du PEFA semble appropriée. Ces contributions comprennent notamment un dialogue politique d'égal à égal ainsi qu'un soutien et des conseils prodigués à long terme au gouvernement partenaire, afin de l'aider à planifier et à mettre en œuvre la réforme ${ }^{22}$. Dans ces pays, l'une des principales tâches de la coopération consiste à identifier des agents du changement et à leur assurer l'appui adéquat ou à leur ménager la marge de manœuvre nécessaire pour faire progresser les réformes de la GFP. Le travail de la coopération ne doit donc pas se limiter à conseiller et à renforcer les exécutifs. Elle doit en effet plutôt contribuer à amorcer les négociations entre gouvernement, administration et société civile.

Les parlements et les acteurs non gouvernementaux ont par ailleurs un rôle clé à jouer dans le contrôle démocratique de la planification des dépenses et de la gestion des finances par l'administration publique. Si les donateurs se doivent certes de renforcer ce rôle, leur tâche est épineuse. En effet, un soutien trop visible ou trop massif risque de miner la crédibilité des groupements de la société civile et de les empêcher d'assumer leur responsabilité dans la GFP. Conscients de ce risque, certains donateurs (Royaume-Uni, Suède et Norvège) soutiennent l'Initiative de la société civile sur le budget (Civil Society Budget Initiative, CSBI), coordonnée par le Projet budgétaire international (IBP) ${ }^{23}$. Dans les pays à faible revenu où les conditions d'une véritable participation de la société civile à la GFP (gouvernance, disponibilité des informations et existence de groupements bien établis dans la société civile) sont remplies, cette initiative accorde un appui financier et technique ciblé aux groupements appropriés, afin de les aider à mener leurs activités dans le domaine de la GFP.

22 Une expérience institutionnelle accumulée au fil du temps par les donateurs constitue une condition préalable essentielle à l'établissement d'un dialogue politique sur la réforme de la GFP. Les changements fréquents parmi les représentants sur place affaiblissent cependant le rôle de conseillers et d'interlocuteurs avisés des donateurs (T. Williamson, «General Budget Support and Public Financial Management Reform: Emerging Lessons from Tanzania and Uganda», in S. Koeberle, Z. Stavreski, and J. Walliser, Budget Support as More Effective Aid? Recent Experiences and Emerging Lessons, Washington, DC, The World Bank, 2006, p. 148).

$23<$ http://www.internationalbudget.org/CSBI.htm>. 
La collaboration avec les parlementaires, en particulier les membres de l'opposition, recèle autant de difficultés que la coopération avec les groupements de la société civile. Les donateurs s'attachent dès lors souvent à soutenir l'administration des parlements ou les commissions parlementaires des finances. A moyen terme, ils se verront néanmoins contraints d'élaborer des programmes et des instruments pour fournir un soutien efficace et ciblé aux parlements et aux acteurs de la société civile dans leur mission en matière de GFP ou de renforcer leur rôle dans ce domaine. Dans une étude récente, l'Overseas Development Institute britannique a dès lors formulé des recommandations pour fournir un appui efficace aux parlements (voir encadré 8).

\section{Encadré 8: Guide pour un renforcement efficace des parlements}

- Répondre à la demande. Le renforcement parlementaire devrait être axé sur la demande et répondre aux besoins locaux plutôt que d'être piloté de l'extérieur.

- S'attaquer aux causes. Le renforcement parlementaire devrait chercher à s'attaquer aux causes des performances insuffisantes et ne pas se contenter de traiter les symptômes.

- Tenir compte du contexte. Le renforcement parlementaire doit pleinement prendre en compte le contexte - y compris le contexte politique - dans lequel fonctionne le parlement.

- Intégrer les bénéficiaires. Le renforcement parlementaire devrait impliquer - outre des membres du gouvernement - diverses organisations locales ou groupes d'intérêts, y compris des parlementaires et des partis de l'opposition.

ـ Partir de problèmes précis. Le renforcement parlementaire devrait partir de problèmes particuliers, tels que le contrôle budgétaire, la lutte contre la corruption, le VIH/sida et la réduction de la pauvreté, afin d'améliorer les performances parlementaires, plutôt que de se concentrer uniquement sur les procédures parlementaires.

- Coordonner et adapter les activités. Les organismes participant au renforcement parlementaire doivent s'efforcer de mieux coordonner leurs activités et s'assurer qu'elles correspondent à l'objectif (renforcer le parlement). II vaut mieux y réfléchir à deux fois avant d'organiser ou de soutenir des visites ou des séminaires d'étude.

ـ Fournir un soutien à long terme.

Source: A. Hudson and C. Wren, Parliamentary Strengthening in Developing Countries, London, Overseas Development Institute (ODI), 2007.

Le besoin de connaissances se fait également sentir dans d'autres domaines de la GFP. Jusqu'ici, le débat international a ainsi réservé trop peu de place aux recettes des budgets publics dans les pays en développement. Si nul ne conteste qu'il est essentiel de renforcer les capacités de mobiliser des ressources indigènes dans les pays en développement, en particulier pour promouvoir la durabilité de la coopération fondée sur les programmes, la plupart des programmes de réforme de la GFP soutenus par les donateurs se concentrent en priorité sur le renforcement de la planification, de la mise en œuvre et du contrôle des dépenses publiques. Bien que la Déclaration de Paris souligne l'importance des recettes locales, même l'approche du PEFA a jusqu'ici négligé ce secteur pourtant crucial de la gestion des finances publiques ${ }^{24}$.

24 Voir: Deutsche Gesellschaft für Technische Zusammenarbeit (GTZ) und KfW, Reform der öffentlichen Finanzen in Entwicklungsländern - Ansatzpunkte für die deutsche EZ, gemeinsame Stellungnahme von GTZ und KfW, Eschborn, GTZ; Frankfurt a.M., KfW, 2005. 
Pour assurer la durabilité de la coopération fondée sur des programmes, les recettes qu'un pays parvient à mobiliser lui-même sont déterminantes à double titre. D'une part, les pays en développement dépendant beaucoup du bon vouloir des donateurs doivent absolument accroître leur capacité d'autofinancement s'ils veulent pouvoir, à moyen ou à long terme, se passer des aides budgétaires et cesser dès lors de dépendre de l'extérieur pour financer les charges courantes de leur budget.

D'autre part, si un pays ne parvient pas à financer ses tâches par le prélèvement d'impôts, de taxes et de redevance, il ne pourra jamais prétendre améliorer la transparence et accroître la responsabilité du gouvernement national envers les citoyens. Et c'est justement dans les pays largement tributaires de l'aide extérieure que les gouvernements n'ont pas encore compris que s'ils sont responsables d'une utilisation des fonds publics propice au développement, c'est moins face aux donateurs que devant la population. Par ailleurs, la plupart des citoyens de ces pays doivent encore prendre davantage conscience de leur pouvoir d'influer sur les institutions et les milieux politiques, afin de les mettre au service de leurs préoccupations (autonomisation). Or, le renforcement de l'efficacité et de la transparence dans l'encaissement de recettes indigènes, dans le cadre de la coopération en matière de GFP, peut grandement contribuer à atteindre ces deux objectifs.

Les connaissances sur les recettes publiques dans les pays en développement font particulièrement défaut dans les domaines suivants: modalités de systèmes fiscaux axés sur le développement et la réduction de la pauvreté lorsque les capacités administratives sont réduites, économie politique de réformes fiscales dans des contextes nationaux spécifiques et formes adéquates d'appui pour les mettre en œuvre.

\section{Encadré 9: Initiative pour la transparence des industries extractives (EITI)}

Dans nombre de pays pourtant riches en ressources naturelles, le développement marque le pas. Ce simple constat témoigne du rôle central de la bonne gouvernance dans le domaine des recettes publiques. L'Initiative pour la transparence des industries extractives (Extractive Industries Transparency Initiative, EITI) représente un progrès important pour remédier à cette situation en appliquant les critères de la bonne gouvernance à la gestion des finances publiques. Lancée par le Royaume-Uni au Sommet de Johannesburg en 2002 et soutenue par le G-8 et la Banque mondiale, cette initiative a permis de formuler 12 principes pour garantir que les recettes provenant des industries extractives serviront à promouvoir le développement durable et la réduction de la pauvreté. Les critères de l'EITI servant à vérifier le respect de ces principes comprennent notamment la publication régulière des montants que les industries pétrolière, gazière et minière versent au gouvernement et le contrôle de ces flux monétaires par des organismes indépendants et satisfaisant aux normes internationales.

Après diverses hésitations, plus de vingt pays ont jusqu'ici déclaré officiellement appliquer les principes de I'EITI. Le principal problème réside dans le fait que le respect de ces principes est volontaire. Plus les "profits imprévus» de l'extraction de matières premières sont élevés, moins les pays tendent à se soumettre aux conventions et aux recommandations, qui ne présentent en fin de compte aucun caractère contraignant. Pour assurer une application stricte de ces principes, la communauté internationale devra donc s'entendre pour définir des normes et des standards contraignants applicables aux entreprises, aux banques, aux investisseurs institutionnels et aux gouvernements, tant du côté des producteurs que du côté des consommateurs.

Sources: <http://www.eitransparency.org>. G. Wurthmann, «3 Fragen an Geerd Wurthmann. Interview mit Geerd Wurthmann", in Deutsche Gesellschaft für Technische Zusammenarbeit (GTZ), Public Finance Reform. Newsletter Öffentliche Finanzen und Verwaltungsreform, Eschborn, GTZ, 2006. 
La même remarque vaut pour la gestion des finances publiques aux niveaux local et régional. Alors que les donateurs soutiennent massivement le processus de décentration dans nombre de pays, le débat sur la politique de développement ne prend pas encore suffisamment en considération la GFP appliquée à ces niveaux. C'est d'autant plus grave que les autorités locales sont amenées à assumer des responsabilités croissantes dans la mise en œuvre des stratégies nationales de développement et de réduction de la pauvreté (SRP), et que l'on cherche à promouvoir l'intégration politique de la population pauvre (souvent rurale) et sa participation aux processus locaux de planification et aux décisions d'allocation des ressources. Si en général on ne parvient pas à coordonner de manière satisfaisante les stratégies nationales de développement et de réduction de la pauvreté avec les activités menées dans ce sens au niveau régional, c'est notamment parce que la GFP n'occupe pas la place qui lui revient dans la plupart des programmes de décentralisation et dans le soutien que la coopération au développement accorde aux administrations locales.

L'intégration d'une perspective genre dans le processus budgétaire va de pair avec l'orientation de la gestion des finances publiques sur la réduction de la pauvreté. Une analyse sexospécifique des finances publiques et l'allocation qui en découle des ressources publiques à divers secteurs et usages ont des répercussions directes sur la répartition des ressources entre les sexes et, partant, sur l'égalité entre les sexes. Bien que 40 pays appliquent déjà l'approche genre à leurs budgets ${ }^{25}$, le sujet ne semble intéresser, en politique de développement, que les réseaux qui se consacrent aux relations sociales entre hommes et femmes et n'a pas reçu l'attention qu'il mérite dans le débat international sur la gestion des finances publiques. Pourtant, comme en témoigne la liste des instruments servant à intégrer la perspective genre dans le processus budgétaire (voir encadré 10), les problèmes liés à l'égalité des sexes et à l'égalité des chances revêtent de l'importance dans tous les secteurs de la politique sociale et à tous les niveaux de la gestion des finances publiques ${ }^{26}$.

\section{Encadré 10:}

\section{Instruments de l'intégration de la perspective genre dans le processus budgétaire}

1. Evaluation des politiques attentive à l'égalité entre les sexes. Elle est conçue pour analyser les politiques et les programmes du point de vue du genre et déterminer dans quelle mesure ces politiques et les ressources qu'on leur alloue sont susceptibles de réduire ou de creuser les inégalités entre les sexes.

2. Evaluation des bénéficiaires ventilée par sexe. Elle est appliquée pour évaluer dans quelle mesure des programmes ou des services répondent aux besoins des bénéficiaires - effectifs ou potentiels tels qu'identifiés et exprimés par ces derniers.

3. Analyse de l'incidence des dépenses publiques, ventilée par sexe. Elle sert à évaluer la répartition des ressources budgétaires entre hommes et femmes, filles et garçons, en estimant les coûts unitaires d'une certaine prestation et en calculant dans quelle mesure les deux groupes utilisent cette prestation.

25 International Budget Project (IBP), Bulletin (bimensuel), n 31, Washington, DC, IBP, 2006.

26 Afin de promouvoir la relation entre les deux tendances du débat autour des budgets soucieux de l'égalité entre hommes et femmes dans la coopération allemande au développement, la Deutsche Gesellschaft für Technische Zusammenarbeit (GTZ, organisme de développement allemand) a publié un manuel de formation sur ce sujet. 
4. Analyse des effets du budget sur I'utilisation du temps, ventilée par sexe. Elle est utilisée pour établir un lien entre l'engagement des dépenses, les prestations qu'elles financent et la façon dont le temps est utilisé par les différents membres d'un ménage.

5. Cadre de politique économique à moyen terme prenant en compte le genre. II permet d'intégrer la perspective genre dans les cadres de référence à moyen terme du développement des politiques, de la planification et de l'exécution du budget, par exemple en ventilant les variables selon le sexe, en combinant les comptes des produits nationaux et les recettes des ménages, ainsi qu'en mettant en évidence et en remettant en question les hypothèses peu soucieuses de la perspective genre sur le fonctionnement de l'économie.

6. Rapport budgétaire prenant en compte le genre. Cet instrument est un rapport établi par des organes gouvernementaux, qui analyse l'implication des dépenses budgétaires sur l'égalité entre hommes et femmes.

7. Analyse de l'incidence de la fiscalité sur les femmes et les hommes. Elle sert à estimer l'écart entre les impacts de la fiscalité sur les femmes et les hommes, ainsi qu'à évaluer le niveau des impôts perçus en rapport avec les besoins et la demande de dépenses publiques.

Source: H. Hofbauer Balmori, Gender and Budgets: Overview Report, Brighton, Institute for Development Studies at the University of Sussex, 2003.

\section{Choix et défis pour la coopération au développement}

\section{Positionnement des donateurs bilatéraux \\ dans le débat international sur la gestion des finances publiques}

Le recours aux instruments diagnostiques en matière de GFP, tel qu'il est prévu par le PEFA ainsi que par le FMI et la Banque mondiale, est bien structuré. Aucune activité bilatérale supplémentaire ne s'impose dans ce domaine. Les divers organismes donateurs devraient plutôt s'attacher à promouvoir l'application des instruments diagnostiques sur place parmi la communauté des donateurs et à exploiter les résultats obtenus pour leurs propres activités.

L'Activité conjointe du CAD (OCDE) sur la gestion des finances publiques offre une plate-forme appropriée pour poursuivre l'élaboration d'efforts conjoints des donateurs, destinés à soutenir les programmes de réforme de la GFP. Cette remarque s'applique également au Réseau du CAD sur la réduction de la pauvreté, au sein duquel la bonne gouvernance (financière) constitue un champ d'activités prioritaire, de même qu'au GOVNET du CAD et à son Groupe de travail sur la fiscalité et la transparence.

\section{Compétences spécifiques et répartition du travail}

Dans les pays qui bénéficient d'un appui essentiellement constitué d'aides budgétaires, il convient de considérer aussi bien les aspects fiduciaires que la politique de développement dans le cadre de la GFP. Dans ces cas, une répartition du travail entre donateurs s'impose d'urgence, afin d'établir et de structurer un dialogue sur l'aide budgétaire aux niveaux macroéconomique et sectoriel, la politique budgétaire formant ici un domaine transversal d'importance.

Les acteurs de la coopération se répartissant le travail selon leurs priorités respectives et la disponibilité de spécialistes en matière de GFP, les donateurs bila- 
téraux ne peuvent pas faire valoir leurs compétences en général, mais seulement dans le contexte du pays considéré. Les problèmes des politiques sectorielles, par exemple dans les secteurs de l'eau et de la santé, apparaissent plus que jamais dans la planification financière à moyen terme de chaque secteur, de même que dans le budget global, qui fixe l'allocation des ressources aux budgets sectoriels. La création d'un profil spécifique à un pays passe donc avant tout par la mobilisation des compétences sectorielles des donateurs présents sur place, afin de promouvoir le dialogue budgétaire.

Il importe d'améliorer les connaissances du personnel de la coopération sur place en matière de gestion des finances publiques, pour accroître les chances des divers donateurs de prendre part de manière équitable au dialogue mené avec les partenaires sur les budgets sectoriels et sur les stratégies de réduction de la pauvreté (SRP), et d'intégrer utilement les compétences sectorielles de leurs divers organes de coopération dans l'établissement de revues des dépenses publiques (PER) et dans la planification de cadres de dépenses à moyen terme (CDMT).

Lors de la planification de réformes de la GFP, il est recommandé, dans les pays dotés d'une SRP ainsi que dans les Etats fragiles, d'aligner les diverses aides sur les programmes de réforme soutenus par la communauté des donateurs. Cela n'implique pas nécessairement une collaboration au programme de réforme financé conjointement, mais un alignement précis de toutes les contributions sur les mesures prévues dans ce programme. Dans d'autres pays bénéficiaires, il s'agit moins d'aligner l'aide sur les activités de la communauté des donateurs que d'identifier les mesures isolées à même de répondre aux besoins des partenaires.

\section{Décentralisation et gestion des finances publiques}

Dans nombre de pays, la décentralisation constitue une priorité, voire une priorité absolue, pour les donateurs. Il est ainsi de plus en plus question de décentralisation dans le domaine de la gouvernance, et les niveaux régional et local tendent à occuper une place prépondérante dans la mise en œuvre des cadres stratégiques de lutte contre la pauvreté (CSLP). Dans un tel contexte, les réformes de la gestion des finances publiques ne peuvent que gagner en importance. Dans la plupart des pays, on ne fait cependant pas suffisamment le lien entre décentralisation et dialogue sur la GFP au niveau macroéconomique.

\section{Recettes publiques et mobilisation de ressources indigènes}

Les mêmes remarques s'appliquent aux recettes des finances publiques. La mobilisation de ressources indigènes appartient aux domaines d'intervention que le débat international sur l'accroissement de l'APD a jusqu'ici négligés. Elle compte pourtant parmi les tâches clés qui garantissent le financement durable du budget. Outre la réforme organisationnelle et le renforcement des capacités dans l'administration fiscale, il importe aussi d'appuyer la mise en place de systèmes fiscaux axés sur la réduction de la pauvreté, tout en tenant compte des moyens de pilotage limités et de l'économie politique propres à chaque pays. 
Le renforcement des cours des comptes dans les pays en développement est l'une des conditions fondamentales pour instaurer la transparence, l'obligation de rendre compte et le contrôle de la société civile sur les activités de l'Etat. Ce faisant, il apporte une contribution essentielle à la bonne gestion gouvernementale, qui dépasse largement le cadre de la seule gouvernance financière, c'està-dire la couverture de risques purement fiduciaires dans le cadre de la GFP. L'expérience a toutefois montré qu'un véritable renforcement des cours des comptes passe par des interventions à différents niveaux. Au niveau micro, il importe de renforcer les capacités de leurs collaborateurs par des cours appropriés de formation et de perfectionnement. Au niveau méso, il faut surtout réformer le développement organisationnel et la gestion du personnel. Au niveau macro, enfin, il convient d'instaurer un vaste dialogue politique, afin de consolider la position de la cour des comptes dans la structure nationale du pouvoir.

\section{Parlements et société civile}

Pour soumettre les fonctions gouvernementales à un véritable contrôle politique et civil, il faut non seulement instituer des cours des comptes indépendantes et dotées du personnel adéquat, mais aussi veiller à ce que les parlements et les groupements de la société civile disposent de capacités suffisantes. Ce sujet étant politiquement très sensible, la plupart des donateurs se sont contentés de renforcer les services parlementaires et ont laissé les organisations non gouvernementales (ONG) se charger de la coopération avec la société civile. Jusqu'ici, la grande majorité des ONG et des fondations politiques n'ont cependant inclus que ponctuellement la gestion des finances publiques dans leurs activités de développement. De plus, les instruments qu'elles mettent en œuvre ne complètent guère les activités de coopération menées par les organismes gouvernementaux pour renforcer les capacités des autorités exécutives en matière de GFP. Il serait donc souhaitable d'intégrer davantage les ONG dans l'élaboration de mécanismes de la gouvernance ayant trait à la GFP dans les pays partenaires. Bien entendu, il ne s'agit nullement d'accroître le degré d'intervention des organismes gouvernementaux de coopération au développement dans le travail des ONG. L'idéal consisterait en fait à densifier le réseau regroupant les divers acteurs et à intensifier leurs contacts et leurs échanges sur la gestion des finances publiques. 


\section{Bibliographie}

Comité d'aide au développement (CAD), Harmoniser l'aide pour renforcer son efficacité, vol. 2: Le soutien budgétaire, les approches sectorielles et le développement des capacités en matière de gestion des finances publiques, Lignes directrices et ouvrages de référence du CAD, Paris, OCDE, 2003.

Department for International Development (DFID), Managing Fiduciary Risks When Providing Poverty Reduction Budget Support, DFID Briefing, London, DFID, 2004.

Dépenses publiques et responsabilité financière (PEFA), Cadre de mesure de la performance de la gestion des finances publiques, Washington, DC, Banque mondiale, 2005.

Dépenses publiques et responsabilité financière (PEFA), Etude des mesures utilisées pour remédier aux lacunes des systèmes de gestion des finances publiques dans le contexte d'un programme d'appui de réformes, Washington, DC, Banque mondiale, 2003.

Deutsche Gesellschaft für Technische Zusammenarbeit (GTZ), Good Financial Governance - Good Governance in Public Finance, Fiscal Studies, n 3, Eschborn, GTZ, 2006.

Deutsche Gesellschaft für Technische Zusammenarbeit (GTZ) und KfW, Reform der öffentlichen Finanzen in Entwicklungsländern - Ansatzpunkte für die deutsche EZ, gemeinsame Stellungnahme von GTZ und KfW, Eschborn, GTZ; Frankfurt a.M., KfW, 2005.

FUNDAR - Centro de Análisis e Investigación, International Budget Project (IBP), and International Human Rights Internship Program (IHRIP), Dignity Counts: A Guide to Using Budget Analysis to Advance Human Rights, México, FUNDAR; Washington, DC, IBP; IHRIP, 2004.

Heller, P., Understanding Fiscal Space, IMF Policy Discussion Paper, nº5/4, Washington, DC, International Monetary Fund, 2005.

Hofbauer Balmori, H., Gender and Budgets: Overview Report, Brighton, Institute for Development Studies at the University of Sussex, 2003.

Hudson, A. and C. Wren, Parliamentary Strengthening in Developing Countries, London, Overseas Development Institute (ODI), 2007.

International Budget Project (IBP), Guide pour répondre au questionnaire sur le budget ouvert. Explicitation des questions et des modalités de réponse, Washington, DC, IBP, 2005.

International Budget Project (IBP), Bulletin (bimensuel), nº 31, Washington, DC, IBP, 2006.

International Development Department (IDD) and Associates, Evaluation of General Budget Support: Synthesis Report, Birmingham, University of Birmingham, 2006.

Leiderer, S., Analyse und Reform des öffentlichen Budgetmanagements : Ein zentrales Querschnittsthema für die programmorientierte Entwicklungspolitik, Analysen und Stellungnahmen, n 3/2005, Bonn, Deutsches Institut für Entwicklungspolitik (DIE), 2005.

Leiderer, S., Öffentliches Budgetmanagement in Entwicklungsländern: Analyseinstrumente und Ansatzpunkte der programmorientierten Entwicklungszusammenarbeit, Berichte und Gutachten, nº 7/2004, Bonn, Deutsches Institut für Entwicklungspolitik (DIE), 2004.

Norton, A. and D. Elson, What's behind the Budget? Politics, Rights and Accountability in the Budget Process, London, Overseas Development Institute (ODI), 2002.

Poverty Reduction and Economic Management (PREM), Fiscal Policy for Growth and Development: An Interim Report, Washington, DC, The World Bank, 2006.

Réseau du CAD sur la gouvernance, Relever le défi posé par le renforcement des capacités. Evoluer vers de bonnes pratiques, Paris, OCDE, 2006.

Robinson, M., Budget Analysis and Policy Advocacy: The Role of Non-governmental Public Action, IDS Working Paper, $n^{\circ} 279$, Brighton, Institute of Development Studies (IDS) at the University of Sussex, 2006.

Shand, D., «Managing Fiduciary Issues in Budget Support Operations », in S. Koeberle, Z. Stavreski, and J. Walliser, Budget Support as More Effective Aid? Recent Experiences and Emerging Lessons, Washington, DC, The World Bank, 2006, pp. 27-44.

Williamson, T., «General Budget Support and Public Financial Management Reform: Emerging Lessons from Tanzania and Uganda», in S. Koeberle, Z. Stavreski, and J. Walliser, Budget Support as More Effective Aid? Recent Experiences and Emerging Lessons, Washington, DC, The World Bank, 2006, pp. 139-152.

Wurthmann, G., «3 Fragen an Geerd Wurthmann. Interview mit Geerd Wurthmann», in Deutsche Gesellschaft für Technische Zusammenarbeit (GTZ), Public Finance Reform. Newsletter Öffentliche Finanzen und Verwaltungsreform, Eschborn, GTZ, 2006. 